\title{
Charge Generation and Mobility-Limited Performance of Bulk Heterojunction Solar Cells with a Higher Adduct Fullerene
}

\author{
Steffen Roland, ${ }^{\dagger}$ Liang Yan, ${ }^{\ddagger}$ Qianqian Zhang, ${ }^{\ddagger}$ (i) Xuechen Jiao," Adrian Hunt, " Masoud Ghasemi,"

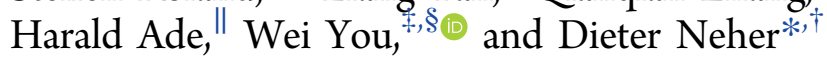 \\ ${ }^{\dagger}$ Institute of Physics and Astronomy, University of Potsdam, Karl-Liebknecht-Str. 24-25, D-14476 Potsdam-Golm, Germany \\ ${ }^{\ddagger}$ Department of Chemistry, University of North Carolina at Chapel Hill, Chapel Hill, North Carolina 27599-3290, United States \\ ${ }^{\S}$ Department of Applied Physical Sciences, CB \#3216, University of North Carolina, Chapel Hill, North Carolina 27599-3216, United \\ States \\ "Department of Physics and Organic and Electronic Laboratory (ORaCEL), North Carolina State University, Raleigh, North Carolina \\ 27695-7518, United States
}

ABSTRACT: Alternative electron acceptors are being actively explored in order to advance the development of bulkheterojunction (BHJ) organic solar cells (OSCs). The indene $-\mathrm{C}_{60}$ bisadduct (ICBA) has been regarded as a promising candidate, as it provides high open-circuit voltage in BHJ solar cells; however, the photovoltaic performance of such ICBA-based devices is often inferior when compared to cells with the omnipresent PCBM electron acceptor. Here, by pairing the high performance polymer (FTAZ) as the donor with either PCBM or ICBA as the acceptor, we explore the physical mechanism behind the reduced performance of the ICBA-based device. Time delayed collection field (TDCF) experiments reveal reduced, yet field-independent free charge

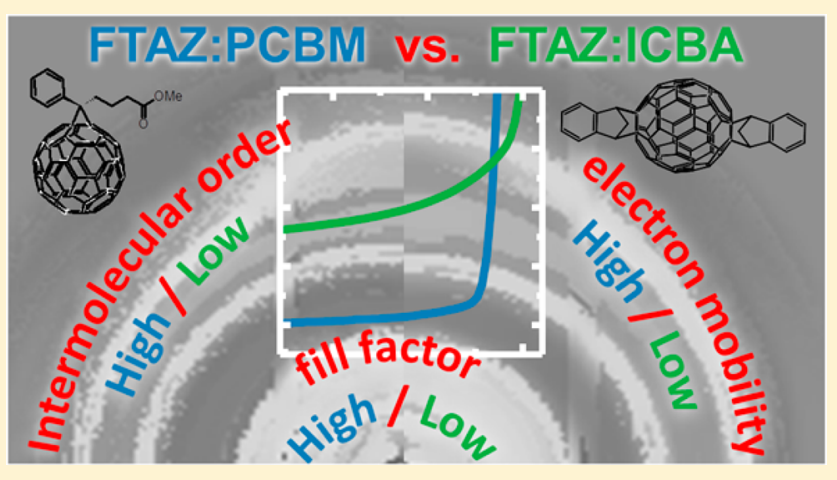
generation in the FTAZ:ICBA system, explaining the overall lower photocurrent in its cells. Through the analysis of the photoluminescence, photogeneration, and electroluminescence, we find that the lower generation efficiency is neither caused by inefficient exciton splitting, nor do we find evidence for significant energy back-transfer from the CT state to singlet excitons. In fact, the increase in open circuit voltage when replacing PCBM by ICBA is entirely caused by the increase in the CT energy, related to the shift in the LUMO energy, while changes in the radiative and nonradiative recombination losses are nearly absent. On the other hand, space charge limited current (SCLC) and bias-assisted charge extraction (BACE) measurements consistently reveal a severely lower electron mobilitiy in the FTAZ:ICBA blend. Studies of the blends with resonant soft X-ray scattering (RSoXS), grazing incident wide-angle X-ray scattering (GIWAXS), and scanning transmission X-ray microscopy (STXM) reveal very little differences in the mesoscopic morphology but significantly less nanoscale molecular ordering of the fullerene domains in the ICBA based blends, which we propose as the main cause for the lower generation efficiency and smaller electron mobility. Calculations of the $J V$ curves with an analytical model, using measured values, show good agreement with the experimentally determined $J V$ characteristics, proving that these devices suffer from slow carrier extraction, resulting in significant bimolecular recombination losses. Therefore, this study highlights the importance of high charge carrier mobility for newly synthesized acceptor materials, in addition to having suitable energy levels.

\section{INTRODUCTION}

The active layer of a polymer solar cell typically consists of a bulk heterojunction $(\mathrm{BHJ})$ blend of one polymer donor and one fullerene acceptor. ${ }^{1}$ The key output characteristics of such a solar cell, including the open circuit voltage $\left(V_{\mathrm{OC}}\right)$, short circuit current $\left(J_{\mathrm{SC}}\right)$, and fill factor $(\mathrm{FF})$, are fundamentally determined by the intricate interplay between charge generation, recombination, and extraction, which is further related to the optical and electronic properties of the polymer donor and the fullerene acceptor in the bulk heterojunction thin film. ${ }^{2,3}$ While the design and synthesis of new polymer donors have been the major driving force in improving the device efficiency, ${ }^{4,5}$ there is only limited progress in engineering the fullerene acceptor. One notable example is the indene $-\mathrm{C}_{60}$ bisadduct (ICBA). ${ }^{6-8}$ Specifically, the higher lying lowest unoccupied molecular orbital (LUMO) of ICBA compared to the LUMO of the ubiquitous phenyl- $\mathrm{C}_{61}$-butyric acid methyl ester (PCBM) generally results in a higher open circuit voltage of ICBA based BHJ solar cells. ${ }^{7}$ If

Received: March 10, 2017

Revised: April 25, 2017

Published: April 26, 2017 
the short circuit current and fill factor of the PCBM:polymer based cells can be maintained when switching to ICBA, the overall energy conversion efficiency $\left(\eta=V_{\mathrm{OC}} \times J_{\mathrm{SC}} \times \mathrm{FF}\right)$ of the ICBA based cells would be significantly improved. This is indeed the case for poly(3-hexylthiophene) (P3HT) based $\mathrm{BHJ}$ solar cells, where swapping PCBM with ICBA led to a $50 \%$ increase in the device efficiency (from 4 to 6.5\%). ${ }^{9}$ However, with other polymers, though a higher $V_{\mathrm{OC}}$ for the ICBA:polymer based photovoltaic device is generally observed, the overall efficiency of the ICBA based device is typically depressed, mainly due to a significantly lower $J_{\mathrm{SC}}$, and often a poor FF as well. ${ }^{10-12}$

Most of the previous studies related the decreased $J_{\mathrm{SC}}$ (and $\mathrm{FF}$ ) to unfavorable energetics. For example, a number of studies have suggested that the driving force for the formation of the charge transfer (CT) state from the initially excited exciton, a key step to the generation of free charge carriers, is not sufficient in ICBA based devices. ${ }^{13-15}$ The driving force of the CT state formation is empirically approximated by the energy difference of the LUMO levels between the donor and the acceptor in organic solar cells for electron transfer, and the difference of the highest occupied molecular orbital (HOMO) levels for hole transfer, respectively. Usually, an energy difference of about $0.2-0.3 \mathrm{eV}$ between the LUMO (HOMO) levels of the donor and the acceptor is considered sufficient for efficient electron (hole) transfer. ${ }^{16,17}$

Following the same line of arguments, Faist et al. correlated the efficiency of exciton quenching and charge generation in polymer/fullerene blend systems to the energy difference $\left(\Delta E_{\mathrm{CS}}\right)$ between the minimal absorption onset energy of the system and its charge transfer state energy $\left(E_{\mathrm{CT}}\right.$ or $\mathrm{CT}$ state energy). ${ }^{10,18}$ It was concluded that ICBA based blends have poorer efficiencies because of a back transfer of energy to the singlet exciton, followed by rapid recombination. ${ }^{11}$ It was also proposed that singlet activation from the CT state constitutes an additional pathway for nongeminate recombination, thereby reducing the fill factor and $V_{\mathrm{OC}}{ }^{18}$ Similar to that, Hoke et al. proposed inefficient hole transfer from ICBA to PBDTTPD (a donor polymer) in combination with efficient energy transfer from PBDTTPD to ICBA, to be responsible for the lower generated current. ${ }^{11}$ Additionally, some literature reports claimed that the recombination loss to the lowest triplet state would also affect the device output of ICBA based blends; ${ }^{11,19,20}$ however, this triplet loss mechanism is still under debate. ${ }^{11}$ In contrast, Albrecht et al. related the efficiency of free charge generation to the energy difference between the CT state and the energy of the charge separated state $\left(E_{\mathrm{CS}}\right)^{12}$ This interpretation was motivated by the observation of similar generation efficiencies when exciting either singlet excitons or CT states, which suggests that the energy of the primary excitation does not determine the efficiency of free charge formation. Other authors reported the electron mobility to be significantly lower in ICBA based devices, ${ }^{8,21}$ which would impede the charge transport and increase the charge recombination, leading to a decreased $J_{\mathrm{SC}}$ and FF.

For some systems, the poorer device performance of the ICBA cell was attributed to an unfavorable morphology. ${ }^{13}$ Pronounced intermixing or even intercalation of the ICBA molecules with the donor molecules or polymers was proposed to result in the localization of the photogenerated electrons on isolated ICBA clusters, favoring geminate recombination and suppressing free charge generation. ${ }^{22}$ Finally, Shoaee et al. explained the lower photogeneration yield of ICBA-based polymer blends to a lower tendency of higher adduct fullerenes to aggregate. ${ }^{23}$ It was argued that ICBA lacks the ability to stabilize photogenerated electrons on the fullerene-rich domains, rendering them more vulnerable to fast geminate recombination. Unfortunately, only few papers combine an in depth study of the photovoltaic properties and the blend morphology, rendering it quite difficult to assign the decrease in efficiency to a specific process.

Here, we study the photovoltaic, photophysical, and morphological properties of bulk heterojunction devices comprising a high performance polymer, PBnDT-FTAZ (abbreviated as FTAZ), blended either with ICBA or PCBM. PBnDT-FTAZ is a well-studied polymer that has shown over $7 \%$ device efficiency in thick active layers when it was blended with PCBM. ${ }^{24-26}$ FTAZ is of particular interest, as it has a similar band gap $(\sim 2.0 \mathrm{eV})$ to that of P3HT but exhibits a significantly larger $V_{\mathrm{OC}}(\sim 0.8 \mathrm{~V})$ in its $\mathrm{BHJ}$ devices due to a lower lying HOMO of about $\sim-5.4 \mathrm{eV}^{24}$ Accordingly, the LUMO level of FTAZ is lower compared to P3HT and the LUMO energy offset to the acceptor is reduced. Importantly, the mesoscopic morphology of polymer-fullerene blends based on FTAZ (and its derivatives) was shown to be quite robust against the variation of the backbone structure. For example, in a study where the degree of backbone fluorination was continuously increased from 25 to $100 \%$ in blends with PCBM, the domain size and purity remained rather unaffected, while subtle changes in the backbone nanoscale molecular packing and orientation led to a vast increase in efficiency. ${ }^{26}$ In a more recent work, the same amount of fluorination was introduced in three distinctly different ways, namely, by employing statistical copolymers of difluorinated and nonfluorinated monomer units, homopolymers with every unit being monofluorinated, and by a 1:1 physical blend of a difluorinated and nonfluorinated polymer. ${ }^{27}$ However, the photovoltaic properties of the three blends with PCBM were virtually the same. We also note that FTAZ blended with PCBM, on account of the mixed domains, was shown to be insensitive to differences in domain size and purities. ${ }^{28}$

Here we show that the $V_{\mathrm{OC}}$ can indeed be further increased to above $1 \mathrm{~V}$ in the FTAZ:ICBA blend (abbreviated as FT:IC) compared to FTAZ:PCBM (abbreviated as FT:PC) but that the FT:IC device suffers from the reduced $J_{\mathrm{SC}}$ and FF, as seen in many other ICBA based systems. To elaborate the reason for the reduced performance, we combined transient and steady state methods to determine recombination losses and mobilities in these two systems. We find that, besides a low charge generation efficiency, the performance of the device is severely limited by insufficient charge extraction due to a low electron mobility. Furthermore, we are able to simulate intensity dependent $J V$ characteristics of the FT:PC and FT:IC devices with a recently introduced modified Shockley equation by Neher et al. ${ }^{29}$ which explicitly considers transport limitations due to low mobilities. Notably, the study of the two blends using advanced X-ray techniques, including resonant soft X-ray scattering (R-SoXS), grazing incident wide-angle X-ray scattering (GIWAXS), and scanning transmission X-ray microscopy (STXM), revealed very little differences in the microscopic and mesoscopic morphology. We conclude that the main reason for the poorer performance of the ICBA-based blend is the inability of ICBA to form domains with high intermolecular order, thereby preventing rapid delocalization and extraction of the photogenerated electrons. 


\section{EXPERIMENTAL RESULTS}

2.1. Device Performance of FTAZ:PCBM versus FTAZ:ICBA. FT:PC and FT:IC samples were fabricated with the following structure: ITO/PEDOT:PSS/FTAZ:fullerene/ $\mathrm{Ca} / \mathrm{Al}$. The active layers were spin coated at University of North Carolina (UNC) and finalized by evaporating the calcium electrode either at the University of Potsdam (UP) or at UNC for comparison. The active layer thickness of all devices was about $110 \mathrm{~nm}$. The photovoltaic performance of FT:PC and FT:IC devices under one sun (AM 1.5 G, $100 \mathrm{~mW}$ $\mathrm{cm}^{-2}$ ) illumination is shown in Figure 1a, with detailed device
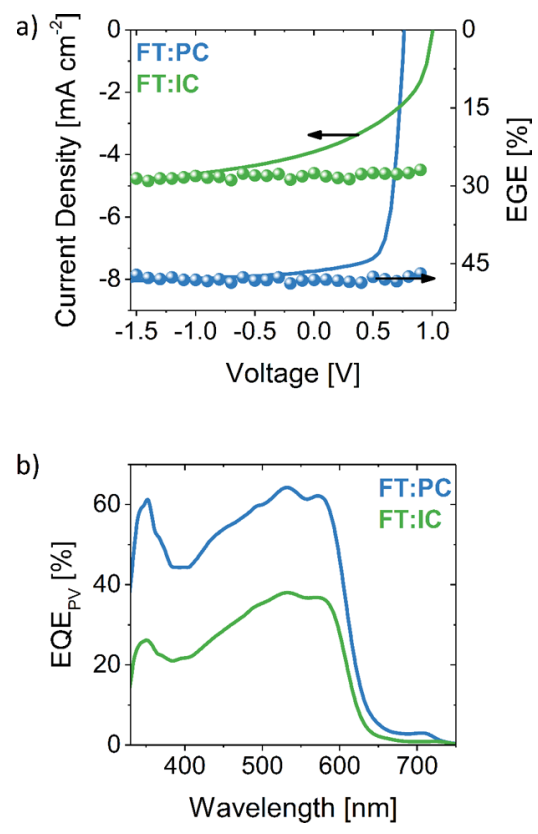

Figure 1. (a) Left axis: $J V$ characteristics of FT:PC and FT:IC solar cells at one sun illumination conditions (AM $1.5 \mathrm{G}, 100 \mathrm{~mW} \mathrm{~cm}^{-2}$ ) at Potsdam. Right axis: External generation efficiency for FT:PC and FT:IC. (b) External quantum efficiency $\left(\mathrm{EQE}_{\mathrm{PV}}\right)$ of FT:PC and FT:IC.

Table 1. Device Parameters of FT:PC and FT:IC Solar Cells

$\begin{array}{ccccc} & J_{\mathrm{SC}}\left(\mathrm{mA} \mathrm{cm}^{-2}\right) & V_{\mathrm{OC}}(\mathrm{V}) & \mathrm{FF}(\%) & \begin{array}{c}\text { efficiency } \\ (\%)\end{array} \\ \text { FT:PC } & 8.6 \pm 0.7 & 0.82 \pm 0.01 & 71.0 \pm 2.0 & 4.9 \pm 0.5 \\ & (8.3 \pm 0.2)^{a} & (0.79 \pm 0.01) & (71.7 \pm 0.9) & (4.7 \pm 0.1) \\ \text { FT:IC } & 4.0 \pm 0.3 & 1.03 \pm 0.01 & 54.0 \pm 2.0 & 2.2 \pm 0.3 \\ & (4.7 \pm 0.2) & (1.02 \pm 0.01) & (53.3 \pm 0.6) & (2.5 \pm 0.1)\end{array}$

${ }^{a}$ The values outside the brackets are measured at University of North Carolina (UNC); the values inside the brackets are measured at University of Potsdam (UP).

characteristics listed in Table 1 . One can clearly observe that the $200 \mathrm{mV}$ higher $V_{\mathrm{OC}}$ of the FT:IC device cannot compensate for the significant drop in both $J_{\mathrm{SC}}$ and FF, leading to an overall reduced device performance of FT:IC ( $\sim 50 \%$ of the device efficiency of FT:PC). Furthermore, exchanging PCBM by ICBA has no apparent effect on the shape of the photovoltaic external quantum efficiency plot (Figure $1 \mathrm{~b}$ ) but results in an overall reduction of the $\mathrm{EQE}_{\mathrm{PV}}$ by a factor of 2 , in line with the noticeably suppressed $J_{S C}$ of the FT:IC device.
2.2. External Quantum Efficiency Spectra and Open Circuit Voltage. Several examples exist where the $V_{\mathrm{OC}}$ of a polymer:fullerene solar cell was increased by exchanging PCBM with ICBA. ${ }^{9,11,30}$ This has been attributed to the electron affinity of ICBA being ca. $0.2 \mathrm{eV}$ lower than that of PCBM; i.e., the LUMO of ICBA is higher than that of PCBM by $\sim 0.2$ $\mathrm{eV} .{ }^{12,18}$ However, recent work showed charge generation and recombination in such devices to proceed through the CT state manifold. ${ }^{31-34}$ As an important consequence, it is the energy of the charge transfer state $\left(E_{\mathrm{CT}}\right)$ rather than the difference between the acceptor LUMO and the donor HOMO which sets the fundamental $V_{\mathrm{OC}}$ limit of organic solar cells. More specifically, $V_{\mathrm{OC}}$ was shown to be given by $E_{\mathrm{CT}} / q$ (with $q$ being the elementary charge) minus the voltage loss due to radiative and nonradiative recombination. Values for the losses can be derived from accurate measurements of the external quantum efficiency spectra of the electroluminescence (EL) and the photogenerated current $\left(\mathrm{EQE}_{\mathrm{EL}}\right.$ and $\left.\mathrm{EQE}_{\mathrm{PV}}\right)$, respectively. ${ }^{31}$

These spectra are shown in Figure 2, together with fits to Gaussian line shapes following a protocol set forth by Vandewal

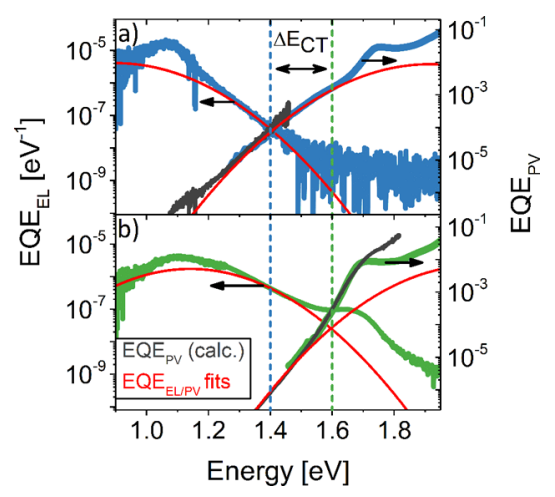

Figure 2. External quantum efficiency of electroluminescence $\left(\mathrm{EQE}_{\mathrm{EL}}\right)$ and the photovoltaic $\mathrm{EQE}_{\mathrm{PV}}$ for (a) FT:PC and (b) FT:IC. The corresponding Gaussian fits are depicted in red, and their crossing point determines the $\mathrm{CT}$ energy. The $\mathrm{EQE}_{\mathrm{PV}}$ calculated from the emission spectra is depicted as a gray line.

et al. ${ }^{31}$ For the FT:PC cells, we can nicely resolve the sub-band gap response in the spectrum of $\mathrm{EQE}_{\mathrm{PV}}$, which originates from the CT absorption (Figure 2a); however, the CT absorption for the FT:IC cell is mostly buried under the absorption of the pure components (Figure $2 \mathrm{~b}$ ). The $\mathrm{EQE}_{\mathrm{EL}}$ is dominated by a broad feature in the NIR for both blends, which we assign to the radiative decay of the CT state. In addition, the FT:IC cell displays a weak emission peaking at about $1.69 \mathrm{eV}$ due to singlet emission from the fullerene. Interestingly, the NIR $\mathrm{EQE}_{\mathrm{EL}}$ spectra are not purely Gaussian in shape; instead, they display an additional narrow emission feature located at nearly the same energy of about $1.1 \mathrm{eV}$ for both blends, albeit more pronounced for the FT:PC blend. At this moment, we are not able to offer a reasonable explanation for this extra emission, but we speculate that it may be caused by interference effects in the device or that it is related to vibronic features of the CT state itself. Fortunately, as we will show later, the presence of this spectral feature has only a weak effect on the accuracy of determining $V_{\mathrm{OC}}$ from the $E_{\mathrm{CT}}$ and the radiative and nonradiative voltage losses.

According to the reciprocity relation derived by Rau, ${ }^{35}$ $\mathrm{EQE}_{\mathrm{EL}}$ is related to $\mathrm{EQE}_{\mathrm{PV}}(\hbar \omega) \propto \mathrm{EQE}_{\mathrm{EL}}(\hbar \omega) / \Phi_{\mathrm{BB}}(\hbar \omega)$, where $\Phi_{\mathrm{BB}}(\hbar \omega)$ is the room temperature blackbody radiation 
Table 2. Energetics and Open Circuit Voltages and the Differences between FT:PC and FT:IC Values Given as $\Delta$

\begin{tabular}{lcccccc} 
& $V_{\mathrm{OC}}(\mathrm{V})$ & $E_{\mathrm{opt}}(\mathrm{eV})$ & $E_{\mathrm{CT}}(\mathrm{eV})$ & $\Delta E(\mathrm{eV}), E_{\mathrm{opt}}-e V_{\mathrm{OC}}$ & $\Delta E_{\mathrm{CS}}(\mathrm{eV}), E_{\mathrm{opt}}-E_{\mathrm{CT}}$ & $\Delta E_{\mathrm{NG}}(\mathrm{eV}), E_{\mathrm{CT}}-e V_{\mathrm{OC}}$ \\
FT:PC & 0.79 & 1.66 & 1.40 & 0.87 & 0.26 & 0.61 \\
FT:IC & 1.00 & 1.64 & 1.60 & 0.64 & 0.04 & 0.60 \\
$\Delta$ & 0.21 & 0.02 & 0.20 & 0.23 & 0.22 & 0.01 \\
\hline
\end{tabular}

flux. This relation holds if charge carrier generation evolves from the same thermalized state manifolds as radiative charge recombination (i.e., charge generation is not dominated by a hot generation process). The gray lines in Figure 2 depict $\mathrm{EQE}_{\mathrm{PV}}$ calculated from the corresponding $\mathrm{EQE}_{\mathrm{EL}}$ spectra. Their shapes fit well to the directly measured $\mathrm{EQE}_{\mathrm{PV}}$, implying that the reciprocity relation holds well in our systems, at least for injection conditions close to $V_{\mathrm{OC}}$. From the best fit of the $\mathrm{EQE}_{\mathrm{PV}}$ and $\mathrm{EQE}_{\mathrm{EL}}$ with Gaussians of the same width, ${ }^{22}$ the $\mathrm{CT}$ state energy of the FT:PC and FT:IC blends is found to be at 1.4 and $1.6 \mathrm{eV}$, respectively. This difference of $0.2 \mathrm{eV}$ between the CT energy of the PCBM and the ICBA device is very similar to the difference in LUMO energy, indicating that the CT binding energy in both blends is nearly identical. Importantly, also the difference in $E_{\mathrm{CT}}$ agrees well with the measured $V_{\mathrm{OC}}$ difference, which implies that the total $V_{\mathrm{OC}}$ loss is independent of the choice of the fullerene.

The fact that both devices display very similar $V_{\mathrm{OC}}$ loss, as listed in Table $2\left(\Delta E_{\mathrm{NG}}\right)$, is quite surprising, given the distinct differences in the EL emission spectra. As mentioned above, the $\mathrm{EQE}_{\mathrm{EL}}$ spectrum of the FT:PC blend displays an extra feature in the NIR at ca. $1.1 \mathrm{eV}$, while FT:IC exhibits additional emission from the fullerene singlet exciton. To address the question to which extent these additional emission features affect the $V_{\mathrm{OC}}$ of our systems, the $V_{\mathrm{OC}}$ was calculated from the $\mathrm{EQE}_{\mathrm{EL}}$ and $\mathrm{EQE}_{\mathrm{PV}}$ spectra by using the relation

$$
\begin{aligned}
V_{\mathrm{OC}} & =\frac{k_{\mathrm{B}} T}{q} \ln \frac{J_{\mathrm{G}}}{J_{0}} \\
& =\frac{k_{\mathrm{B}} T}{q} \ln \frac{J_{\mathrm{G}}}{J_{0, \mathrm{rad}}}+\frac{k_{\mathrm{B}} T}{q} \ln \left(\mathrm{EQE}_{\mathrm{EL}, \text { int }}\right) \\
& =V_{\mathrm{OC}, \mathrm{rad}}-\Delta V_{\mathrm{OC}, \text { nonrad }}
\end{aligned}
$$

Here, $V_{\mathrm{OC}, \text { rad }}$ is the open circuit voltage in the case of purely radiative recombination, $\Delta V_{\mathrm{OC} \text {,nonrad }}$ is the reduction of the open circuit voltage by additional nonradiative recombination, $k_{\mathrm{B}} T$ is the thermal energy, $q$ is the elementary charge, $J_{0}$ is the dark recombination current (including radiative and nonradiative recombination), and $\mathrm{EQE}_{\mathrm{EL} \text {,int }}$ is the spectrally integrated external quantum efficiency for EL emission. $\mathrm{EQE}_{\mathrm{EL} \text {,int }}$ is also defined as the ratio of the radiative dark recombination current $J_{0 \text {,rad }}$ and the total dark recombination current $J_{0}$. The radiative dark recombination current $J_{0, \operatorname{Rad}}$ according to Rau, can be calculated from the $\mathrm{EQE}_{\mathrm{PV}}$ spectrum by

$$
J_{0, \mathrm{rad}}=q \int_{0}^{\infty} \mathrm{EQE}_{\mathrm{PV}}(\hbar \omega) \Phi_{\mathrm{BB}}(\hbar \omega) \mathrm{d} \hbar \omega
$$

To calculate $J_{0, \text { rad }}, \operatorname{EQE}_{\mathrm{PV}}(\hbar \omega)$ in eq 2 was either used as measured (combining the directly measured $\mathrm{EQE}_{\mathrm{PV}}$ with the one obtained from $\mathrm{EQE}_{\mathrm{EL}}$ via the reciprocity relation; see the gray lines in Figure 2), or it was approximated by combining the measured $\mathrm{EQE}_{\mathrm{PV}}$ with a single broad Gaussian for the low energy part of the spectrum (red lines in Figure 2, neglecting any spectrally narrow feature). The modeled spectra and the values for the radiative limits obtained therefrom are shown in Supporting Information Figure S1 and Table S1, respectively. Noticeably, these two approaches give values for $V_{\mathrm{OC}, \mathrm{rad}}$ which differ by at most $20 \mathrm{mV}$. Notably, also the influence of the singlet emission on the $V_{\mathrm{OC}}$ of the FT:IC blend is very small. This is mainly due to its small overall contribution to the EL emission but also to its higher energy of this narrow emission feature. Therefore, recombination via the singlet state does not constitute a significant channel for $V_{\mathrm{OC}}$ losses in our studied blends. To complete the analysis, we also determined the nonradiative $V_{\mathrm{OC}}$ loss via eq 1 , with $\mathrm{EQE}_{\mathrm{EL} \text {,int }}$ obtained from the integration of the full experimental $\mathrm{EQE}_{\mathrm{EL}}$ spectrum. The results, summarized in Table $S 1$, reveal similar values for both systems, highlighting the rather small effect of the additional spectrally narrow emission features on the overall dark recombination current.

We emphasize that it was not possible to drive the ICBA blend at a condition without showing the notable singlet emission (as shown in Figure 2) in the $\mathrm{EQE}_{\mathrm{EL}}$ spectra. The peak of emission of ICBA at $\sim 1.65 \mathrm{eV}$ is visible even for very low currents $\left(0.28 \mathrm{~mA} \mathrm{~cm}{ }^{-2}\right)$, injected at $0.94 \mathrm{~V}$ forward bias, which is even slightly below open circuit conditions. Electroluminescence from fullerene singlet states in similar polymer:fullerene blend systems was seen by others before, and was shown to be related to a small value of $\Delta E_{\mathrm{CS} \cdot}{ }^{11,18}$ It has been reasoned that singlet emission arises from direct injection of holes into the HOMO of ICBA, which becomes more likely at high injection currents. ${ }^{11}$ This process is, however, unlikely to occur at injection conditions close to $V_{\mathrm{OC}}$, which are used in this study. Rather, the emission observed at $V_{\mathrm{OC}}$, or even slightly below $V_{\mathrm{OC}}$, indicates that an equilibrium between $\mathrm{CT}$ and fullerene singlet states is established. Though this equilibrium might reduce the generation of free charge carriers by opening an additional geminate recombination channel, ${ }^{18}$ our results show that it is less important for the nonradiative recombination of charge and the corresponding $V_{\mathrm{OC}}$ loss.

To finalize this section, we separate the total loss between the absorption onset of the blend $\left(E_{\mathrm{opt}}\right)$ and the $V_{\mathrm{OC}}$ into $\Delta E_{\mathrm{CS}}$ and $\Delta E_{\mathrm{NG}}$ according to Faist and co-workers. ${ }^{18}$ Here, $\Delta E_{\mathrm{CS}}=E_{\mathrm{opt}}-$ $E_{\mathrm{CT}}$ is regarded as the exciton separation energy and $\Delta E_{\mathrm{NG}}=$ $E_{\mathrm{CT}}-e V_{\mathrm{OC}}$ is the nonradiative and radiative losses as discussed above. ${ }^{18,31}$ Table 2 summarizes all relevant energy levels and the measured $V_{\mathrm{OC}}$ values, together with the absolute difference $\Delta$ between all determined values. The difference in $V_{\mathrm{OC}}$ is almost entirely due to the increase of CT state energy for FT:IC compared to FT:PC cells, which is predominantly determined by the difference in LUMO energy of the two fullerene acceptors. The nongeminate losses of $\approx 0.6 \mathrm{eV}$ are similar in both blends, indicating similar steady state nongeminate recombination for FT:PC and FT:IC.

2.3. Generation of Free Charge Carriers. The lower $\mathrm{EQE}_{\mathrm{PV}}$ and the associated smaller $J_{\mathrm{SC}}$ of the FT:IC cell suggest a likely cause: less efficient charge carrier generation. Furthermore, the low FF of the FT:IC cell is indicative of a more pronounced field dependence of charge generation. To address this point in detail, time delayed collection field (TDCF) 
Table 3. SCLC Mobilities and Effective Mobilities

\begin{tabular}{cccc} 
mobility $\left(\times 10^{-4} \mathrm{~cm}^{2} \mathrm{~V}^{-1} \mathrm{~s}^{-1}\right)$ & FT:PC & FT:IC & PCBM \\
\hline electron $^{a}$ & $5.5 \pm 3.4$ & $0.25 \pm 0.11$ & ICBA \\
hole $^{a}$ & $3.4 \pm 1.2$ & $3.4 \pm 1.2$ & $0.8 \pm 0.3$ \\
electron $^{b}$ & $4.2 \pm 0.1$ & $0.14 \pm 0.04$ & $3.1 \pm 0.1$ \\
hole $^{b}$ & $4.3 \pm 0.9$ & $0.37 \pm 0.2$ \\
effective $^{c}$ & $3.1 \pm 0.8$ & $0.27(0.47)$ & \\
effective $^{d}$ & $4.2(4.2)$ & &
\end{tabular}

was employed to measure the external generation efficiency (EGE) of free charge photogeneration as a function of electric field for application-relevant carrier densities. ${ }^{12,26,36}$ Here, charge carriers are generated at a given prebias $V_{\text {pre }}$ by illumination with a short laser pulse at a wavelength of $532 \mathrm{~nm}$, followed by rapid extraction with a reverse voltage pulse $\left(V_{\text {coll }}=\right.$ $-3 \mathrm{~V})$. For short delays and a sufficiently large reverse bias, nongeminate recombination of photogenerated charge is negligible. The time resolution of our TDCF setup has recently been improved, and charges are extracted about 4-6 ns after laser excitation. ${ }^{37}$ Thus, with the known fluence of the laser excitation, EGE is determined as a function of prebias. We emphasize the importance of providing a very short delay time between charge generation and extraction, as fast nongeminate recombination might lower the total amount of extractable charges even after a few nanoseconds. The effect of a fast nongeminate recombination has recently been investigated to greater detail by Kurpiers et al. ${ }^{37}$

Figure 1 presents the voltage dependence of the EGE (measured at a fluence of $0.2 \mu \mathrm{J} \mathrm{cm}^{-2}$ and with a delay of $6 \mathrm{~ns}$ ), together with the $J V$ characteristics of the FT:IC cell and the FT:PC cell. Two important conclusions emerge from these data. First, the EGE of the FT:PC solar cell is 40\% larger than that of the FT:IC cell, matching the ratio of the steady state currents for these two cells at 1 sun condition (AM 1.5 G, 100 $\mathrm{mW} \mathrm{cm} \mathrm{cm}^{-2}$ ) under reverse bias. Therefore, insufficient charge generation is indeed the main cause for the overall lower current of the ICBA blend. Second, we found EGE to be field independent in both cells, despite the large FF difference. This means that the reason for the poorer performance of the cell with ICBA must not lie in a more Coulombically bound precursor state (such as a more tightly bound CT state), as this would split more easily into free charges when applying a high enough field, resulting in a field dependence of free charge generation. Rather than that, replacing ICBA by PCBM results in an overall smaller number of formed and/or extractable free charges.

As briefly discussed in the Introduction, a low current output has been observed for several ICBA containing solar cells and different reasons have been put forward to explain this result. One possible reason may be a too low exciton separation energy $\Delta E_{\mathrm{CS}}$, which is only $0.04 \mathrm{eV}$ for FT:IC compared to $0.26 \mathrm{eV}$ in FT:PC (Table 1). In general, a critical, systemdependent threshold value of $\Delta E_{\mathrm{CS}}$ for efficient charge generation has been reported in many polymer fullerene blends, ranging between $0.26 \mathrm{eV}$ and up to $0.81 \mathrm{eV} .^{10,18}$ Below that value, distinct emission of the fullerene singlet state was often observed and correlated to a reduced photocurrent generation. ${ }^{10,18}$ However, PL measurements on a neat FTAZ layer and the blends reveal similar PL quenching regardless of whether ICBA or PCBM was added to the polymer; thus, inefficient singlet exciton harvesting is not the reason for the significantly lower EGL of FT:IC compared to FT:PC (Figure S8). Alternatively, back transfer of energy from the CT state to strongly bound singlet excitons has been proposed to compete with free carrier formation. ${ }^{18}$ As shown above, our EL studies suggest the formation of singlet excitons from the CT state, but the contribution of singlet formation and recombination to the overall recombination rate seems to be quite small. Therefore, the actual cause for the small EGE can neither be inefficient CT formation from photogenerated excitons nor efficient geminate recombination of $\mathrm{CT}$ states via back-formation of singlet excitons. Another explanation may arise from morphological differences compared to PCBM based cells. A picture derived from transient absorption spectroscopy (TAS) studies on blends of either PCBM or ICBA with small molecules revealed a significant first order charge recombination loss in the ICBA based blend at early times. ${ }^{22}$ It was previously proposed that the ICBA based blend comprises small ICBA islands (i.e., the acceptor phase) embedded into the donor phase; electrons, captured by these islands, would undergo rapid quasi-first-order recombination with mobile holes and reduce the number of extractable free charges. ${ }^{22}$ Notably, we observe a marked decrease in the EGE when increasing the delay time between pulsed illumination and charge extraction in the TDCF experiment, as can be seen in Supporting Information Figure S2, and this decrease becomes more prominent with decreasing internal field (more positive bias). This early decay of the density of extractable carriers, observed only in the ICBAcontaining device, might indeed be reminiscent of such an initial quasi-first-order recombination loss.

2.4. Charge Extraction and Non-Geminate Recombination. Given the field independent generation of charges in both blends, the difference in fill factor must lie in a less favorable balance between extraction and nongeminate recombination. If charge carriers are not sufficiently extracted, the likelihood of recombination increases as the bimolecular recombination rate depends quadratically on the charge carrier density present in the device. For example, we previously showed that, for solar cells comprised of FTAZ:PCBM and the nonfluorinated version HTAZ:PCBM, a FF decrease in the HTAZ:PCBM based device could be explained by less efficient charge carrier extraction due to a reduced hole mobility in the HTAZ:PCBM based device. ${ }^{26}$ Additionally, the bimolecular recombination process itself might be more efficient, represented by a larger bimolecular recombination coefficient.

Thus, we next applied space charge limited current (SCLC) measurements to determine electron and hole mobilities for both FT:IC and FT:PC blends, at both places (UP and UNC). In Figure S4, typical SCLCs (measured at UP) are shown for electron and hole only devices of FT:PC and FT:IC. The SCLC data measured at UNC are shown in Figures S5-S7. 
The mean mobility values, extracted from at least three different pixels, are summarized in Table 3. It has been argued that mobility values might differ when samples are measured in different laboratories. ${ }^{38}$ Also, all samples measured at UP were shipped from UNC without top electrodes, meaning that the active layer was rather unprotected. Despite these differences, the SCLC mobilities determined at both UP and UNC are in good agreement within their respective errors.

To complement these studies, an effective mobility $\left(\mu_{\text {eff }}\right)$ was determined on the actual bipolar devices with bias assisted charge extraction (BACE) following the routine as outlined by Albrecht et al. ${ }^{39}$ Here, the carrier density is measured in two ways: either as a function of voltage for a given (constant) illumination intensity or as a function of the open circuit voltage as the illumination intensity increases. Measured charge carrier density data and the $J V$ characteristics at different illumination conditions are shown in Figure 3. Black symbols are for the

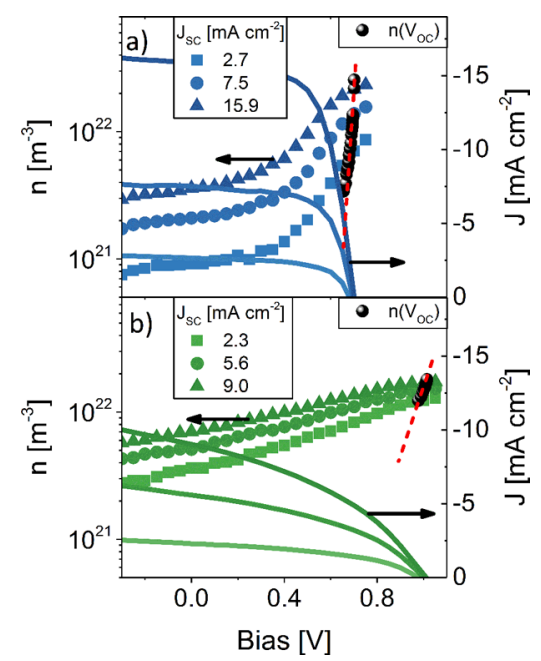

Figure 3. BACE data for (a) FT:PC and (b) FT:IC. The collected charge carrier density, the $J V$ characteristics for the same illumination condition, and the extracted charges at open circuit conditions for different illumination intensities are displayed.

carrier densities measured at $V_{\mathrm{OC}}$ with increasing illumination intensity, while colored symbols show the carrier density as a function of voltage for a constant illumination intensity. The corresponding current densities are plotted with colored lines. While carrier densities at $V_{\mathrm{OC}}$ are very similar for both systems for comparable illumination intensities, they drop much faster with reducing voltage in the FT:PC blend, indicating that charge extraction is more efficient. From those data, an effective mobility was calculated following the model outlined by Albrecht et al., ${ }^{39}$ where $\mu_{\text {eff }}$ is related to the electron and hole mobility, $\mu_{\mathrm{e}}$ and $\mu_{\mathrm{h}}$, by

$$
\mu_{\mathrm{eff}}=\frac{2 \mu_{\mathrm{e}} \mu_{\mathrm{h}}}{\mu_{\mathrm{e}}+\mu_{\mathrm{h}}}
$$

Effective mobilities were determined for three different illumination intensities chosen in a voltage range between -0.3 $\mathrm{V}$ and the $V_{\mathrm{OC}}$ of either FT:PC or FT:IC. The dependence of mean effective mobilities on the applied external voltage is depicted in Figure S3, and the mean mobility values close to open circuit conditions are listed in Table 3. The measured effective mobilities are in good agreement with the effective mobilities calculated according to eq 4 from the SCLC single carrier mobilities. This agreement supports that mobilities measured on unipolar electron- and hole-only devices are representative for mobilities in the bipolar blend, despite the different electrodes used in these electron- or hole-only devices. Small differences in the effective mobilities from BACE and SCLC measurements can be ascribed to the slight degradation of the FT:PC cell, which usually occurred after the transient TDCF measurements and before the BACE measurements. The impact of the minor degradation to the measurements can be seen from the slightly lower $V_{\mathrm{OC}}$ (Figure 3a) than taken from the $J V$ characteristics, measured directly after preparation and before the transient experiments. Most importantly, there is consistent evidence that the electron mobility in the FT:IC device is at least 1 order of magnitude lower than that in the FT:PC device. Note that previous work on thick FTAZ:PCBM blends (ca. $350 \mathrm{~nm}$ ) revealed overall larger electron and hole mobilities. ${ }^{26}$ In the present study, much thinner active layers of ca. $110 \mathrm{~nm}$ were employed, as ICBA devices suffer from a severe reduction of performance for thicker layers.

To estimate whether the low electron mobility in FT:IC is intrinsically limited by the transport capability of the ICBA, electron-only devices of ITO/PEI/pure PCBM or ICBA/Ca/Al were analyzed in the SCLC regime (Figure S7). These measurements indeed reveal a much poorer electron mobility of the pure ICBA layer than that of PCBM, consistent with earlier studies on related fullerene compounds. ${ }^{40}$ In the blend with FTAZ, the electron mobility is further reduced by a factor of $2-3$, indicating that electron transport is also disrupted by (likely) insufficient percolation.

The precise measurement of the carrier density at $V_{\mathrm{OC}}$ with BACE further allows us to evaluate the rate of steady state, nongeminate recombination. With the generation current taken from the reverse bias current at the same illumination intensity and assuming bimolecular recombination with the rate $R=k_{2} n^{2}$, we determined the bimolecular recombination coefficient $k_{2}$ at $V_{\mathrm{OC}}$ and one sun illumination conditions $\left(\approx 1 \times 10^{22} \mathrm{~m}^{-3}\right.$, Figure 3) to be $1.8 \times 10^{-17}$ and $2.1 \times 10^{-17} \mathrm{~m}^{3} \mathrm{~s}^{-1}$ for the FT:IC and FT:PC solar cell, respectively. The very similar bimolecular recombination coefficients are in accordance with the comparable $V_{\mathrm{OC}}$ losses for the two blends. More importantly, the similar bimolecular recombination coefficients indicate that the reduced FF of the FT:IC cell is predominantly caused by the lower effective extraction mobility, which is in turn due to a 1 order of magnitude lower electron mobility in the FT:IC cell compared to the FT:PC device.

2.5. Morphology Investigation. As pointed out above, the lower generation rate in ICBA-based blends had previously been attributed to an intimately intermixed blend morphology. ${ }^{22,23}$ We therefore utilized transmission resonant soft X-ray scattering (R-SoXS) to provide highly quantitative and statistical information about the lateral morphology of our active layers. ${ }^{41}$ Analogous to small-angle X-ray scattering which relies on electron density differences between composite materials, R-SoXS also probes lateral morphology but benefits from enhancing scattering intensity by tuning the X-ray energy to the absorption edge of constituent elements. This allows for significantly improved interpretation of the resulting spectra based on the total integrated scattering intensity (ISI), which contains information about domain spacing and is sensitive to domain contrast and therefore is a measure of domain purity. ${ }^{42}$ It has been well established that the relative average composition variation of blends (or relative domain purity in the two-phase model, where the overall volume fraction of all 
phases is constant) is proportional to the square root of the total integrated scattering intensity. ${ }^{43,44}$

The Lorentz-corrected, thickness, absorption, and contrast normalized (see Figure S9 for details) 1-D R-SoXS scattering profiles are displayed in Figure 4. Both the FT:PC and FT:IC

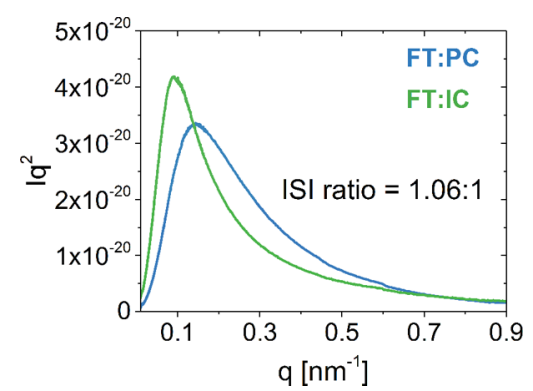

Figure 4. Lorentz-corrected, thickness, absorption, and contrastnormalized 1D R-SoXS profiles of FT:PC and FT:IC at $283.8 \mathrm{eV}$.

blends were measured at $283.8 \mathrm{eV}$ as the resonant incident $\mathrm{X}$ ray energy, where the scattering from mass-thickness variation is minimized and the scattering from compositional variation is maximized. ${ }^{42}$ In Figure 4, the scattering profiles of FT:PC and FT:IC blends exhibit a similar shape with slightly shifted apparent peak position. As indicated in Figure 4, the ISI ratio between FT:PC and FT:IC is 1.06:1, implying nearly identical relative average composition variation between $\mathrm{FT}: \mathrm{PC}$ and FT:IC blends. We can therefore safely rule out a higher degree of intermixing in the ICBA-based blends.

Additionally, the domain spacing is extracted as the characteristic mode length scale, calculated using $d=2 \pi$ / $q_{\text {peak }}$ where $q_{\text {peak }}$ is the peak location of the scattering intensity.
It follows that the domain spacing is $46 \mathrm{~nm}$ for FT:PC and 69 $\mathrm{nm}$ for FT:IC, meaning that exchanging PCBM by ICBA in the blend with FTAZ actually results in more coarse-grained phase separation. With comparable domain purities, smaller domain spacing usually induces higher $J_{S C}$, as a result of larger interfacial area between donor-rich and acceptor-rich domains. ${ }^{45}$ However, the only slightly reduced domain spacing of FT:PC, compared to FT:IC, is not sufficient to contribute to the 2-fold increase of $J_{\mathrm{SC}}$ observed in FT:PC. Furthermore, FT:PC is relatively insensitive to differences in domain spacing and purities and can give very high performance even with domains much larger than observed here. ${ }^{28}$ We surmise that this insensitivity to domain spacing is also operable here. Thus, other morphological factors, particular relating to the intermolecular order on the local scale, must be considered to fully understand the superior performance of FT:PC over FT:IC.

Hence, GIWAXS was conducted on FT:PC and FT:IC with the emphasis toward the molecular packing and ordering of PCBM vs ICBA. Parts a and $c$ of Figure 5 plot the 1D GIWAXS profiles along various azimuthal angles from in-plane $\left(0^{\circ}\right)$ to out-of-plane $\left(90^{\circ}\right)$ direction. The actual GIWAXS profiles of the pure materials are shown in the Supporting Information (Figure S10). From these profiles, it is evident that FTAZ aggregates preferentially adopt a face-on orientation with respect to the substrate in pure thin film and retain this preferential orientation even when blended with PCBM or ICBA (Figure 5a and c). Besides, the fullerene coherence length is also extracted, following the Scherer equation, $c=2 \pi / \mathrm{fwhm}$, where fwhm is the full width at half-maximum of the X-ray diffraction peak. As summarized in Table 4, PCBM is revealed to have a coherence length of $24.3 \AA$ in pure film, while it remains around $22.9 \AA$ in the FT:PC blend. On the other hand,

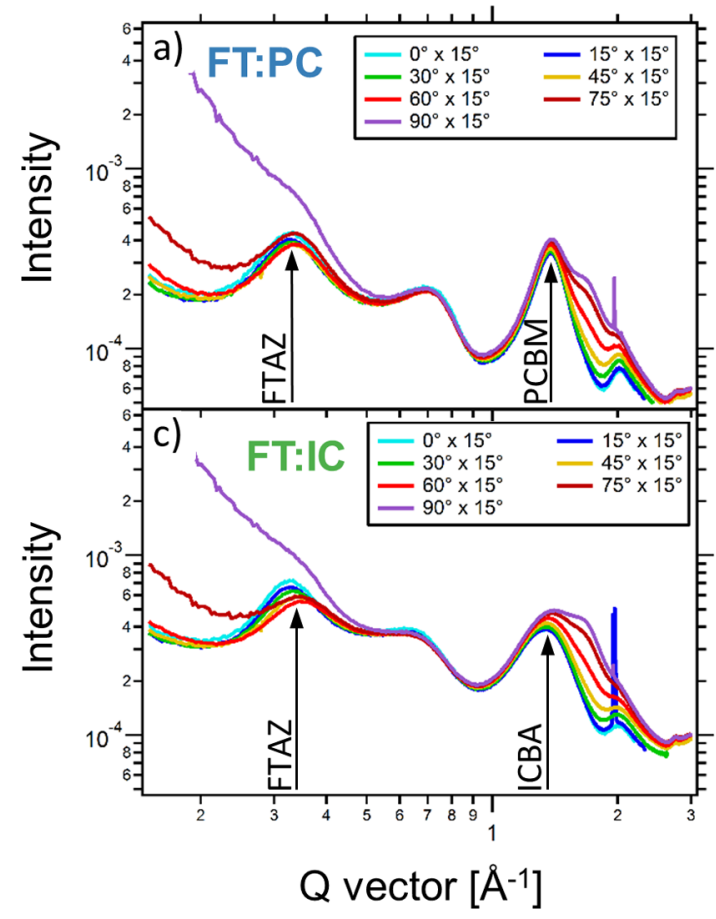

b)

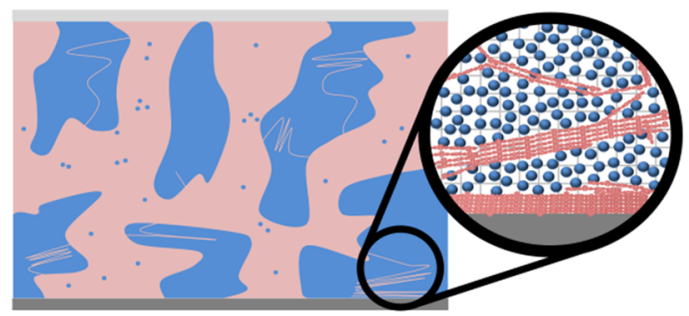

d)

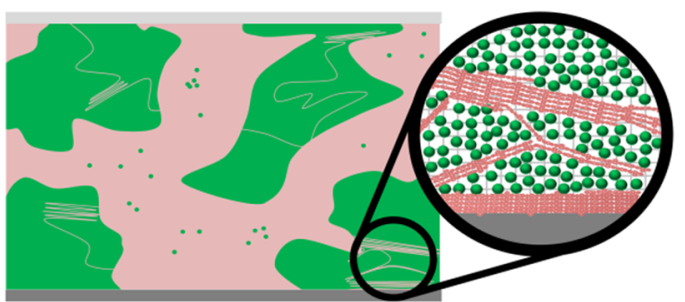

Figure 5. 1D GIWAXS profiles of (a) FT:PC and (c) FT:IC along varying azimuthal angles from $0^{\circ}$ (in-plane) to $90^{\circ}$ (out-of-plane). In parts b and $\mathrm{d}$, simplified sketches of the FT:PC and FT:IC mesoscale morphology are depicted. The sketches are based on all morphology data and not just the GIWAXS profiles presented in parts a and c. The domain size and purity inferred from R-SoXS are similar. The domain spacing of FT:IC is slightly larger than that in FT:PC. The enlarged sections indicate the preferentially face-on orientation of FTAZ in both systems. 
Table 4. Coherence Length of Polymer and Fullerene

\begin{tabular}{cccccc} 
& pure FTAZ & pure PCBM & pure ICBA & FT:PC & FT:IC \\
coherence length of fullerene $(\AA)$ & N/A & $24.3 \pm 0.1$ & $18.5 \pm 0.3$ & $22.9 \pm 2.8$ & $15.2 \pm 0.8$ \\
coherence length of polymer ${ }^{a}(\AA)$ & $63.0 \pm 0.4$ & N/A & N/A & $50.6 \pm 1.3$ & $48.7 \pm 2.9$ \\
${ }^{a}$ The coherence length of polymer is calculated from the in plane $(100)$ peak. & & & \\
\hline
\end{tabular}

pure ICBA thin film only exhibits a coherence length of around 18.5 $\AA$, which even reduces to $15.2 \AA$ in the FT:IC blend. The more than $30 \%$ reduced coherence length of ICBA in FT:IC, compared with PCBM in FT:PC, largely deteriorates the electron mobility in FT:IC.

To better understand the aggregation and crystallization behaviors of PCBM clusters and ICBA clusters within the bulkheterojunction active layers and possibly the difference in coherence length, isothermal crystallization was conducted on FT:PC and FT:IC blends. The as-cast blends were annealed on hot plates in $\mathrm{N}_{2}$ atmosphere at 100,140 , and $170{ }^{\circ} \mathrm{C}$ for 3 days, respectively. STXM was utilized to provide quantitative and chemical sensitive images in real space with a high resolution up to $50 \mathrm{~nm} .{ }^{46}$ While clear PCBM crystals can be observed from FT:PC across all annealing temperatures, complete absence of ICBA crystal is found from all FT:IC samples (see Figure S11 for details). Furthermore, differential scanning calorimetry (DSC) measurements as shown in Figure S12 clearly reveal melting peaks from pure PCBM and FT:PC annealed at varying temperatures, whereas no melting peaks can be seen in the DSC scans from either pure ICBA or FT:IC. This suggests an intrinsic lack of crystallizability of ICBA, indicating that the molecular interactions of these two fullerenes are rather different. It is thus not surprising that the ordering of amorphous aggregates observed with WAXS shows reduced ordering for ICBA compared to PCBM.

\section{DISCUSSION OF THE BLEND MORPHOLOGY IN RELATION TO PHOTOVOLTAIC PARAMETERS}

In Figure $5 b$ and $d$, we offer simplified sketches of the morphologies for the FT:PC and FT:IC devices, respectively. Importantly, the overall morphology of the FTAZ blend turned out to be robust toward an exchange of the fullerenes. We had previously observed a similar behavior when blending the low bandgap polymer Si-PCPDTBT with four different fullerenes, and assigned this to the strong tendency of the polymer to aggregate. $^{12}$

For FTAZ based BHJ blends, exchanging PCBM by ICBA affects domain spacing and composition only very little. This explains the similar PL quenching efficiencies in FT:PC and FT:IC. Also, the choice of the fullerene has virtually no effect on the packing and orientation of the polymer backbones. Given the high sensitivity of the vertical carrier mobility on the orientation of the backbone, ${ }^{47}$ this explains why hole mobilities exhibit very similar mobilities in the two blends. Also, the FTAZ (100) diffraction peaks are at exactly the same positions in the two blends, ruling out extensive polymer-fullerene intercalation. We also believe that these structural similarities provide the reason for the very similar $V_{\mathrm{OC}}$ loss. According to work by Vandewal and Burke, ${ }^{31,34}$ this loss is a function of various parameters, notably the volume fraction of mixed phase, the CT lifetime, and the energetic disorder at the interface. Though we cannot access all of these parameters separately, our morphological data allow us to rule out severe differences in the blend composition at the mesoscopic scale. Then, comparable $V_{\mathrm{OC}}$ losses in both blends point to similar electronic structures and local environment of CT states formed with either PCBM and ICBA, which is understandable given the very small difference in polymer chain packing.

The main difference in morphology is the intermolecular order in the fullerene-rich domains, which we propose to be the decisive parameter when considering the photovoltaic performances of the two blends. Our data show that FT:IC lacks crystallizability and ordering in the fullerene aggregates. In accordance to this, while hole mobilities in FT:PC and FT:IC are comparable, the electron mobility in FT:IC is 1 order of magnitude lower than that in FT:PC. It was suggested that the PCBM coherence length controls the electron mobility. ${ }^{48,49}$

Poor molecular ordering and low electron mobility might also be the reasons for the lower charge generation efficiency in the ICBA blend. Recent work by Stolterfoht et al. presented a striking correlation between the efficiency for free charge generation and the mobility of the slower carrier. ${ }^{50}$ Also, recent TAS studies by Jakowetz et al. on blends of two polymers with higher adduct fullerenes revealed a significant decrease of the charge transfer rate across the donor/acceptor interface in combination with a more pronounced signal in pump-pushphotocurrent $(\mathrm{PPPc})$ experiments compared to the respective PCBM blends. ${ }^{51}$ By considering blends with different polymer:fullerene mixing ratios, these authors came to the conclusion that mainly the size of the fullerene aggregate and its intermolecular order, and not so much the energetic driving force, determine the efficiency of charge generation. Notably, Shoaee and co-workers pointed out that the low photogeneration efficiency in blends consisting of ICBA can be explained by consideration of relative energetics of dispersed matrix vs aggregated domains. ${ }^{23}$ In PCBM systems comprising both mixed polymer/PCBM and aggregated PCBM, they observed that aggregated PCBM films exhibit an electron affinity approximately $100 \mathrm{meV}$ greater than PCBM dispersed in a polymer matrix. This difference in electron affinity was suggested to provide a cascade of energy to localize free charges in the aggregated domains, thus spatially separating the charges. However, in the ICBA blends, due to the lack of aggregation, no such energy offset is present, thus resulting in rapid recombination of charges. These studies suggest that aggregation and ordering is a requirement for efficient stabilization of charges.

\section{SIMULATION OF THE JV CHARACTERISTICS WITH MEASURED PARAMETERS}

At this point, we have identified the lower electron mobility and, possibly related to this, the lower generation efficiency in the FT:IC devices, when compared to the FT:PC cells, to be an important reason for the much poorer performance of the ICBA based device. In order to confirm that less efficient generation and extraction accounts for most of the performance reduction in the FT:IC devices, measured $J V$ characteristics are compared with $J V$ curves calculated with a recently developed analytical model. ${ }^{29}$ According to this model, the $J V$ curves of a solar cell (in the absence of strong space charge effects) can be approximated by 

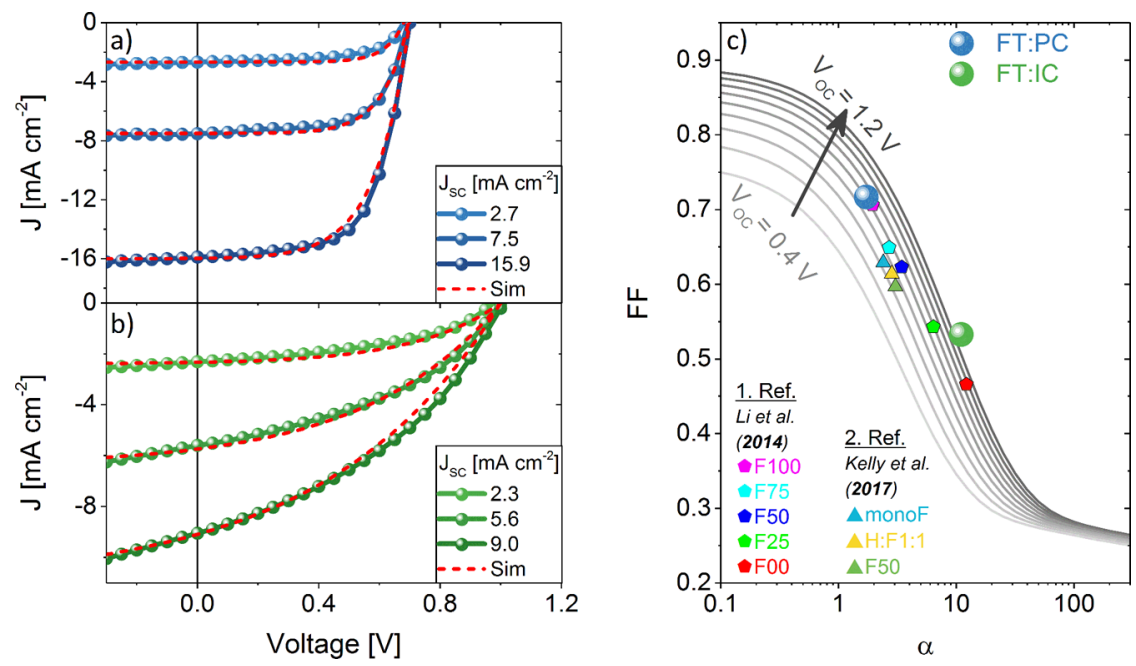

Figure 6. JV characteristics of (a) FT:PC and (b) FT:IC cells illuminated with different intensities of a blue laser diode used for the BACE measurements. The red dashed lines are the simulations according to eq 5. In part c, the fill factor is plotted as a function of the corresponding alpha value. Full lines denote the model prediction according to Neher et al. ${ }^{29}$ for $V_{\mathrm{OC}}$ values ranging from 0.4 to $1.2 \mathrm{~V}$. The FT:PC and FT:IC data are shown as big spheres. Additionally, reference data are plotted which are taken from our previous work including FTAZ. The first reference (1. Ref.) ${ }^{26}$ compared solar cells comprising PCBM as acceptor and different block copolymers containing different amounts of fluorinated monomer units (F100, all fluorinated and F00 none fluorinated). The second reference (2. Ref.) ${ }^{27}$ investigated the effect of different ways of incorporating $50 \%$ fluorination in the donor compound.

$$
J=J_{\mathrm{G}}\left\{\exp \left[\frac{q}{k_{\mathrm{B}} T(1+\alpha)}\left(V_{\mathrm{ext}}-V_{\mathrm{OC}}\right)\right]-1\right\}
$$

Here, a figure of merit $(\alpha)$ is used to account for the competition between free charge recombination and charge extraction:

$$
\alpha=\sqrt{\frac{q k_{2} d^{3} J_{\mathrm{G}}}{4 \mu_{\mathrm{eff}}^{2}\left(k_{\mathrm{B}} T\right)^{2}}}
$$

with $J_{\mathrm{G}}$ being the photogeneration current density, $d$ the device thickness, $k_{2}$ the steady state bimolecular recombination coefficient, $q$ the elementary charge, $\mu_{\mathrm{e} / \mathrm{h}}$ the electron/hole mobility, $k_{\mathrm{B}}$ the Boltzmann constant, and $T$ the temperature. ${ }^{29}$ It was shown that devices exhibit Shockley-type transport characteristics for $\alpha<1$, while they become severely transport limited for $\alpha>1$.

Parts a and b of Figure 6 compare $J V$ curves of the devices used in the BACE measurements (as depicted in Figure 3), for three different illumination intensities, with the corresponding analytical predictions, using recombination coefficients and effective mobilities as determined in the previous section from the BACE measurements. The photogeneration current density is set to values extracted under reverse bias conditions. For both blends, we have observed an excellent agreement between the measured and predicted $J V$ curves. This observation indicates that the device can consistently be described by charge extraction in competition with bimolecular recombination, and that the performance of the FT:IC device is severely hampered by the low electron mobility. In fact, $\alpha$ for the FT:IC device and AM 1.5 G illumination is ca. 12, compared to ca. 2 for FT:PC, highlighting the significant transport limitation in the ICBA-based blend. In general, a high FF can only be achieved when $\alpha$ is low. In Figure 6c, the FF is depicted as a function of $\alpha$ for both systems together with the predictions according to Neher et al. ${ }^{29}$ (shown as gray lines for different $V_{\mathrm{OC}}$ values) and $\mathrm{FF}(\alpha)$ values calculated from parameters that

were presented in other publications on similar systems, which all exhibited similar recombination coefficients. ${ }^{26,27}$ The measured dependency of $\operatorname{FF}(\alpha)$ follows the prediction for the FT:PC and FT:IC devices. The severe transport limitation in FT:IC due to a low electron mobility is reflected in a high value for $\alpha$ and concomitantly a low FF.

\section{CONCLUSION}

We show that the inferior performance of FT:IC compared to FT:PC cells can unequivocally be explained by a lower generation efficiency of free charges, together with a lower extraction efficiency due to a severely decreased electron mobility in the FT:IC device. The low efficiency of both charge carrier generation and extraction cannot compensate for the increase in $V_{\mathrm{OC}}$ by ca. $0.2 \mathrm{~V}$ when going from PCBM to ICBA, leading to the significantly lower device efficiency of the FT:IC solar cell. Electroluminescence and $\mathrm{EQE}_{\mathrm{PV}}$ measurements of the two blends reveal that the difference in $V_{\mathrm{OC}}$ exactly corresponds to the shift in the CT energy, meaning that $V_{\mathrm{OC}}$ losses due to radiative and nonradiative recombination are nearly identical in both systems. EL measurements of FT:IC indicate that singlet excitons on the pure components are reformed by energy transfer from the CT state but that this process seems to have very little effect on the open circuit voltage losses, meaning that reformation of singlet excitons from the CT state does not constitute a significant additional loss channel. In accordance to this, BACE experiments reveal similar nongeminate recombination rates at $V_{\mathrm{OC}}$ for both blends. We also show that the hole mobilities are similar for both blends but that the electron mobility in FT:IC is about 1 order of magnitude smaller than that in FT:PC. By modeling the $J V$ curves of both cells with an analytical model that considers the competition between charge extraction and bimolecular recombination, we are able to prove that the lower effective mobility in the FT:IC device indeed explains the smaller fill factor of this device. Thereby, we identify inefficient electron extraction as a major cause for the low efficiency of the 
FTAZ:ICBA device. Studies of the mesoscale morphology with advanced X-ray techniques show very little changes of the domain size and purity, and on the orientation and packing of the polymer chains, while they reveal a significantly smaller coherent length in the ICBA aggregates indicating less intermolecular ordering. We propose that the commonly observed decrease in the device performance when exchanging PCBM by higher adduct fullerenes has its morphological origin in a poorer nanoscale, intermolecular order of the electrontransporting fullerene phase.

\section{EXPERIMENTAL SECTION}

Device Fabrication and Thin Film Preparation. Glass substrates coated with patterned indium doped tin oxide (ITO) were purchased from Lumtec. The substrates were ultrasonicated in deionized water, acetone, and then 2-proponal for $15 \mathrm{~min}$ each. The substrates were dried under a stream of nitrogen and subjected to treatment by UV-ozone for $15 \mathrm{~min}$. A dispersion of PEDOT:PSS in water (Clevios AI4083 from Heraeus) was filtered by a $0.45 \mu \mathrm{m}$ PVDF filter, then spun-cast onto cleaned ITO substrates at $4000 \mathrm{rpm}$ for $60 \mathrm{~s}$, and then baked at $120{ }^{\circ} \mathrm{C}$ for $15 \mathrm{~min}$ in air to give a thin film with a thickness of $40 \mathrm{~nm}$. Blends of polymer: $\mathrm{PC}_{61} \mathrm{BM}(1: 2 \mathrm{w} / \mathrm{w}, 7$ $\mathrm{mg} / \mathrm{mL}$ for polymer) were dissolved in 1,2,4-trichlorobenzene with heating at $130{ }^{\circ} \mathrm{C}$ for $6 \mathrm{~h}$. All of the solutions were filtered through a $1.0 \mu \mathrm{m}$ poly(tetrafluoroethylene) (PTFE) filter and spun-cast at an optimized rpm for 1 min onto the PEDOT:PSS layer. The substrates were transferred into a vacuum chamber immediately after spin-coating and then dried at $30 \mathrm{mmHg}$ below atmosphere for $30 \mathrm{~min}$. The devices were finished for measurement after thermal deposition of a $10 \mathrm{~nm}$ film of calcium and a $100 \mathrm{~nm}$ aluminum film as the cathode at a base pressure of $2 \times 10^{-6} \mathrm{mbar}$. For the devices fabricated at UNC, there are 8 cells per substrate, each with an active area of 13 $\mathrm{mm}^{2}$ per device. The cells finalized at UP had pixels with either 16 or $1.1 \mathrm{~mm}^{2}$ for spectral analysis or transient measurements, respectively. Device characterization was carried out under AM $1.5 \mathrm{G}$ irradiation with an intensity of $100 \mathrm{~mW} \mathrm{~cm} \mathrm{~cm}^{-2}$ (Oriel 91160, $300 \mathrm{~W}$ ) calibrated by a NREL certified standard silicon cell. Current density versus voltage $(J V)$ curves were recorded with a Keithley 2400 digital source meter. $\mathrm{EQE}_{\mathrm{PV}}$ was detected under monochromatic illumination (OrielCornerstone monochromator in combination with a $200 \mathrm{~W}$ halogen lamp), and the calibration of the incident light was performed with a UV enhanced silicon photodiode calibrated by Newport (Newport 818-UV) or a germanium photodiode (Newport 818-IR). All fabrication steps after adding the PEDOT:PSS layer onto the ITO substrates and characterizations were performed in gloveboxes under a nitrogen atmosphere. The final performance average values and standard deviations are deduced from eight devices.

Device Physics: (BACE, TDCF, SCLC, Electroluminescence Spectra). TDCF: ${ }^{52}$ Pulsed excitation from a diode pumped, Q-switched Nd:YAG laser (NT242, EKSPLA) with 6 ns pulse duration and $500 \mathrm{~Hz}$ repetition rate was used to generate charges in the device. A pulse generator (Agilent $81150 \mathrm{~A}$ ) was used to apply the pre- and collection bias in combination with a home-built amplifier. The current through the samples was measured via a $50 \Omega$ resistor and recorded with an oscilloscope (Yokogawa DL9140). The pulse generator was triggered with a fast photodiode (EOT, ET-2030TTL).

BACE: ${ }^{39}$ The same setup as for TDCF is used, except for the illumination conditions. Here we illuminate with a high power
$1 \mathrm{~W}, 445 \mathrm{~nm}$ laser diode (insaneware) with a switch off time of about $10 \mathrm{~ns}$. The LED is operated at $500 \mathrm{~Hz}$ with a duty cycle of $90 \%$ of one period. This realizes $1.8 \mathrm{~ms}$ of illumination before the diode is switched off for $200 \mu \mathrm{s}$. By this measurement routine, steady state conditions are established. After switching the light off, the voltage at the sample is reversed and all charges are extracted. The extraction voltage was set to $-3 \mathrm{~V}$ to ensure extraction of all charges in the device.

SCLC mobility (at UNC and UP): Hole mobility was acquired through the hole-only devices with a configuration of ITO/PEDOT:PSS/FTAZ:acceptor/ $\mathrm{MoO}_{3} / \mathrm{Al}$. Electron mobility was acquired through the electron-only devices with the configuration of ITO/PEI/FTAZ:acceptor/Ca/Al, with a hole blocking layer of polyethylenimine (PEI). The experimental dark current densities of FTAZ:acceptor blends were measured for applied voltages from 0 to $5 \mathrm{~V}$. The hole injection was from the $\mathrm{MoO}_{3}$ side for the hole-only device, and the electron injection was from the Ca side for the electron-only device. The applied voltage $V$ was corrected from the voltage drop $V_{\mathrm{rs}}$ across the series resistance and contact resistance of indium tin oxide/poly(3,4-ethylene-dioxythiophene):poly(styrenesulfonic acid) (ITO/PEDOT:PSS) for the hole-only device or ITO/PEI for the electron-only device, which is found from a reference device without the active polymer layer. The built-in voltage is assumed as $0 \mathrm{~V}$. From the plots of $J^{0.5}$ vs $V$, hole or electron mobilities of FTAZ:acceptor blends can be deduced from the equation

$$
J=\frac{9}{8} \varepsilon_{\mathrm{r}} \varepsilon_{0} \mu \frac{V^{2}}{L^{3}}
$$

where $\varepsilon_{0}$ is the permittivity of free space, $\varepsilon_{\mathrm{r}}$ is the dielectric constant of the polymer which is assumed to be around 3 for the conjugated polymers, $\mu$ is the mobility, $V$ is the voltage drop across the device, and $L$ is the film thickness of the active layer.

Electroluminescence: The EL spectra were acquired with an Andor SR393i-B spectrometer equipped with a silicon detector DU420ABR-DD and an InGaAs DU491A-1.7 detector. Spectra were collected for different injection currents with both detectors and normalized. The normalized spectra were scaled to absolute values by a separate calibration measurement, where the absolute photon flux was measured with a calibrated silicon photodiode for the same injection conditions.

Resonant soft X-ray scattering: R-SoXS measurements were performed at beamline 11.0.1.2 in Advanced Light Source (ALS), Lawrence Berkeley National Laboratory. ${ }^{41}$ FT:PC and FT:IC thin films were spin-cast on top of PEDOT:PSS with an identical procedure as the device fabrication, followed by being floated via deionized water. The samples were investigated under high vacuum $\left(1 \times 10^{-7}\right.$ Torr $)$ in order to reduce the absorption of soft X-rays in air.

Scanning transmission X-ray microscopy (STXM) and near edge X-ray absorption fine structure (NEXAFS): STXM and NEXAFS measurements were performed at beamline 5.3.2.2 in Advanced Light Source (ALS), Lawrence Berkeley National Laboratory. ${ }^{46}$ A $240 \mu \mathrm{m}$ diameter zone plate with an outermost zone-width of $25 \mathrm{~nm}$ was used. The entrance and exit slits were at 50 and $25 \mu \mathrm{m}$, respectively. Approximately 0.6 Torr of $\mathrm{N}_{2}$ was bled into a section of the beamline to improve spectral purity. 


\section{ASSOCIATED CONTENT}

\section{S Supporting Information}

The Supporting Information is available free of charge on the ACS Publications website at DOI: 10.1021/acs.jpcc.7b02288.

$\mathrm{EQE}_{\mathrm{EL}}$ spectra (Figure S1); determination of the radiative limit from fitted $\mathrm{EQE}_{\mathrm{EL}}$ spectra (Figure $\mathrm{S} 1$ ); delay dependent EGE data (TDCF) (Figure S2); effective mobility by BACE (Figure S3); SCLC data (Figures S4-S7); photoluminescence and absorption spectra (Figure S8); scattering contrast function (Figure S9); GIWAXS profiles of the pure materials (Figure S10); STXM images of isothermally annealed FT:PC and FT:IC at varying temperatures (Figure S11); thermal properties of PCBM, ICBA, FT:PC, and FT:IC by DSC scans (Figure S12) (PDF)

\section{AUTHOR INFORMATION}

\section{Corresponding Author}

*E-mail: neher@uni-potsdam.de.

ORCID

Steffen Roland: 0000-0002-8299-5560

Qianqian Zhang: 0000-0002-8896-1708

Wei You: 0000-0003-0354-1948

\section{Notes}

The authors declare no competing financial interest.

\section{ACKNOWLEDGMENTS}

D.N. and S.R acknowledge financial support by the German Ministry of Science and Education (Project UNVEIL, FKZ 13N13719). W.Y. and L.Y. thank the National Science Foundation for funding (Grant: NSF ECCS-1344745) and Dr. Bhoj R. Gautam and Prof. Kenan Gundogdu at NCSU for the assistance of PL measurement. W.Y. and Q.Z. thank the National Science Foundation and the Office of Naval Research for funding (Grant: NSF DMR-1507249 and ONR N000141410221). The DSC instrument acquisition and DSC measurements by Masoud Ghasemi were supported by a Research Opportunity Initiative grant by the UNC General Administration. X.J., A.H., and H.A. gratefully thank the support by the ONR grant N00141512322. X-ray data acquired at beamlines 11.0.1.2., 7.3.3, and 5.3.2.2 at the ALS in Berkeley National Lab, which was supported by the U.S. Department of Energy (DE-AC02-05CH11231).

\section{REFERENCES}

(1) Nelson, J. Polymer:Fullerene Bulk Heterojunction Solar Cells. Mater. Today 2011, 14, 462-470.

(2) Bartesaghi, D.; Perez, I. d. C.; Kniepert, J.; Roland, S.; Turbiez, M.; Neher, D.; Koster, L. J. A. Competition between Recombination and Extraction of Free Charges Determines the Fill Factor of Organic Solar Cells. Nat. Commun. 2015, 6, 7083.

(3) Mauer, R.; Howard, I. A.; Laquai, F. J. Phys. Chem. Lett. 2010, 1, $3500-3505$.

(4) Zhou, H.; Yang, L.; You, W. Rational Design of High Performance Conjugated Polymers for Organic Solar Cells. Macromolecules 2012, 45, 607-632.

(5) Xiao, S.; Zhang, Q.; You, W. Molecular Engineering of Conjugated Polymers for Solar Cells: An Updated Report. Adv. Mater. 2016, 1601391.

(6) Xin, H.; Subramaniyan, S.; Kwon, T. W.; Shoaee, S.; Durrant, J. R.; Jenekhe, S. a. Enhanced Open Circuit Voltage and Efficiency of Donor-Acceptor Copolymer Solar Cells by Using Indene-C60 Bisadduct. Chem. Mater. 2012, 24, 1995-2001.
(7) He, Y.; Chen, H.-y.; Hou, J.; Li, Y. Indene-C(60) Bisadduct: A New Acceptor for High-Performance Polymer Solar Cells. J. Am. Chem. Soc. 2010, 132, 1377-1382.

(8) Huang, J. H.; Hsiao, Y. S.; Richard, E.; Chen, C. C.; Chen, P.; Li, G.; Chu, C. W.; Yang, Y. The Investigation of Donor-Acceptor Compatibility in Bulk-Heterojunction Polymer Systems. Appl. Phys. Lett. 2013, 103, 043304.

(9) Zhao, G.; He, Y.; Li, Y. 6.5\% Efficiency of Polymer Solar Cells Based on Poly(3-Hexylthiophene) and Indene-C60 Bisadduct by Device Optimization. Adv. Mater. 2010, 22, 4355-4358.

(10) Faist, M. A.; Shoaee, S.; Tuladhar, S.; Dibb, G. F. A.; Foster, S.; Gong, W.; Kirchartz, T.; Bradley, D. D. C.; Durrant, J. R.; Nelson, J. Understanding the Reduced Efficiencies of Organic Solar Cells Employing Fullerene Multiadducts as Acceptors. Adv. Energy Mater. 2013, 3, 744-752.

(11) Hoke, E. T.; Vandewal, K.; Bartelt, J. A.; Mateker, W. R.; Douglas, J. D.; Noriega, R.; Graham, K. R.; Fréchet, J. M. J.; Salleo, A.; McGehee, M. D. Recombination in Polymer:Fullerene Solar Cells with Open-Circuit Voltages Approaching and Exceeding 1.0 V. Adv. Energy Mater. 2013, 3, 220-230.

(12) Albrecht, S.; Vandewal, K.; Tumbleston, J. R.; Fischer, F. S. U.; Douglas, J. D.; Fréchet, J. M. J.; Ludwigs, S.; Ade, H.; Salleo, A.; Neher, D. On the Efficiency of Charge Transfer State Splitting in Polymer:Fullerene Solar Cells. Adv. Mater. 2014, 26, 2533-2539.

(13) Kang, T. E.; Cho, H. H.; Cho, C. H.; Kim, K. H.; Kang, H.; Lee, M.; Lee, S.; Kim, B.; Im, C.; Kim, B. J. Photoinduced Charge Transfer in Donor-Acceptor ( $\mathrm{Da})$ Copolymer: Fullerene Bis-Adduct Polymer Solar Cells. ACS Appl. Mater. Interfaces 2013, 5, 861-868.

(14) Cheng, P.; Li, Y.; Zhan, X. Efficient Ternary Blend Polymer Solar Cells with Indene-C60 Bisadduct as an Electron-Cascade Acceptor. Energy Environ. Sci. 2014, 7, 2005-2011.

(15) Coffey, D. C.; Larson, B. W.; Hains, A. W.; Whitaker, J. B.; Kopidakis, N.; Boltalina, O. V.; Strauss, S. H.; Rumbles, G. An Optimal Driving Force for Converting Excitons into Free Carriers in Excitonic Solar Cells. J. Phys. Chem. C 2012, 116, 8916-8923.

(16) Dennler, G.; Scharber, M. C.; Brabec, C. J. Polymer-Fullerene Bulk-Heterojunction Solar Cells. Adv. Mater. 2009, 21, 1323-1338.

(17) Scharber, M. C. M. C.; Mühlbacher, D.; Koppe, M.; Denk, P.; Waldauf, C.; Heeger, a. J. A. J.; Brabec, C. J. C. J. Adv. Mater. 2006, 18, 789-794.

(18) Faist, M. A.; Kirchartz, T.; Gong, W.; Ashraf, R. S.; McCulloch, I.; de Mello, J. C.; Ekins-Daukes, N. J.; Bradley, D. D. C.; Nelson, J. Competition between the Charge Transfer State and the Singlet States of Donor or Acceptor Limiting the Efficiency in Polymer:Fullerene Solar Cells. J. Am. Chem. Soc. 2012, 134, 685-692.

(19) Schlenker, C. W.; Chen, K.-S.; Yip, H.-L.; Li, C.-Z.; Bradshaw, L. R.; Ochsenbein, S. T.; Ding, F.; Li, X. S.; Gamelin, D. R.; Jen, A. K. Y.; et al. Polymer Triplet Energy Levels Need Not Limit Photocurrent Collection in Organic Solar Cells. J. Am. Chem. Soc. 2012, 134, 19661-19668.

(20) Rao, A.; Chow, P. C. Y.; Gélinas, S.; Schlenker, C. W.; Li, C.-Z.; Yip, H.-L.; Jen, A. K. Y.; Ginger, D. S.; Friend, R. H. The Role of Spin in the Kinetic Control of Recombination in Organic Photovoltaics. Nature 2013, 500, 435-439.

(21) Miller, N. C.; Sweetnam, S.; Hoke, E. T.; Gysel, R.; Miller, C. E.; Bartelt, J. a.; Xie, X.; Toney, M. F.; McGehee, M. D. Molecular Packing and Solar Cell Performance in Blends of Polymers with a Bisadduct Fullerene. Nano Lett. 2012, 12, 1566-1570.

(22) Ko Kyaw, A. K.; Gehrig, D.; Zhang, J.; Huang, Y.; Bazan, G. C.; Laquai, F.; Nguyen, T.-Q. High Open-Circuit Voltage Small-Molecule P-Dts(Fbtth2)2:Icba Bulk Heterojunction Solar Cells - Morphology, Excited-State Dynamics, and Photovoltaic Performance. J. Mater. Chem. A 2015, 3, 1530-1539.

(23) Shoaee, S.; Subramaniyan, S.; Xin, H.; Keiderling, C.; Tuladhar, P. S.; Jamieson, F.; Jenekhe, S. A.; Durrant, J. R. Charge Photogeneration for a Series of Thiazolo-Thiazole Donor Polymers Blended with the Fullerene Electron Acceptors Pcbm and Icba. Adv. Funct. Mater. 2013, 23, 3286-3298. 
(24) Price, S. C.; Stuart, A. C.; Yang, L. Q.; Zhou, H. X.; You, W. Fluorine Substituted Conjugated Polymer of Medium Band Gap Yields $7 \backslash \%$ Efficiency in Polymer-Fullerene Solar Cells. J. Am. Chem. Soc. 2011, 133, 4625-4631.

(25) Li, W.; Yang, L.; Tumbleston, J. R.; Yan, L.; Ade, H.; You, W. Controlling Molecular Weight of a High Efficiency Donor-Acceptor Conjugated Polymer and Understanding Its Significant Impact on Photovoltaic Properties. Adv. Mater. 2014, 26, 4456-4462.

(26) Li, W.; Albrecht, S.; Yang, L.; Roland, S.; Tumbleston, J. R.; McAfee, T.; Yan, L.; Kelly, M. A.; Ade, H.; Neher, D.; et al. MobilityControlled Performance of Thick Solar Cells Based on Fluorinated Copolymers. J. Am. Chem. Soc. 2014, 136, 15566-15576.

(27) Kelly, M. A.; Roland, S.; Zhang, Q.; Lee, Y.; Kabius, B.; Wang, Q.; Gomez, E. D.; Neher, D.; You, W. Incorporating Fluorine Substitution into Conjugated Polymers for Solar Cells: Three Different Means, Same Results. J. Phys. Chem. C 2017, 121, 20592068.

(28) Tumbleston, J. R.; Stuart, A. C.; Gann, E.; You, W.; Ade, H. Fluorinated Polymer Yields High Organic Solar Cell Performance for a Wide Range of Morphologies. Adv. Funct. Mater. 2013, 23, 34633470.

(29) Neher, D.; Kniepert, J.; Elimelech, A.; Koster, L. J. A. A New Figure of Merit for Organic Solar Cells with Transport-Limited Photocurrents. Sci. Rep. 2016, 6, 24861.

(30) Di Nuzzo, D.; Wetzelaer, G.-J. A. H.; Bouwer, R. K. M.; Gevaerts, V. S.; Meskers, S. C. J.; Hummelen, J. C.; Blom, P. W. M.; Janssen, R. A. J. Simultaneous Open-Circuit Voltage Enhancement and Short-Circuit Current Loss in Polymer: Fullerene Solar Cells Correlated by Reduced Quantum Efficiency for Photoinduced Electron Transfer. Adv. Energy Mater. 2013, 3, 85-94.

(31) Vandewal, K.; Tvingstedt, K.; Gadisa, A.; Inganäs, O.; Manca, J. V. Relating the Open-Circuit Voltage to Interface Molecular Properties of Donor:Acceptor Bulk Heterojunction Solar Cells. Phys. Rev. B: Condens. Matter Mater. Phys. 2010, 81, 125204.

(32) Vandewal, K.; Tvingstedt, K.; Gadisa, A.; Inganas, O.; Manca, J. V. On the Origin of the Open-Circuit Voltage of Polymer-Fullerene Solar Cells. Nat. Mater. 2009, 8, 904-909.

(33) Vandewal, K.; Albrecht, S.; Hoke, E. T.; Graham, K. R.; Widmer, J.; Douglas, J. D.; Schubert, M.; Mateker, W. R.; Bloking, J. T.; Burkhard, G. F.; et al. Efficient Charge Generation by Relaxed ChargeTransfer States at Organic Interfaces. Nat. Mater. 2014, 13, 63-68.

(34) Burke, T. M.; Sweetnam, S.; Vandewal, K.; McGehee, M. D. Beyond Langevin Recombination: How Equilibrium between Free Carriers and Charge Transfer States Determines the Open-Circuit Voltage of Organic Solar Cells. Adv. Energy Mater. 2015, 5, 1500123.

(35) Rau, U. Reciprocity Relation between Photovoltaic Quantum Efficiency and Electroluminescent Emission of Solar Cells. Phys. Rev. B: Condens. Matter Mater. Phys. 2007, 76, 085303.

(36) Kurpiers, J.; Balazs, D. M.; Paulke, A.; Albrecht, S.; Lange, I.; Protesescu, L.; Kovalenko, M. V.; Loi, M. A.; Neher, D. Free Carrier Generation and Recombination in Pbs Quantum Dot Solar Cells. Appl. Phys. Lett. 2016, 108, 103102.

(37) Kurpiers, J.; Neher, D. Dispersive Non-Geminate Recombination in an Amorphous Polymer:Fullerene Blend. Sci. Rep. 2016, 6, 26832.

(38) Blakesley, J. C.; Castro, F. A.; Kylberg, W.; Dibb, G. F. A.; Arantes, C.; Valaski, R.; Cremona, M.; Kim, J. S.; Kim, J.-S. Towards Reliable Charge-Mobility Benchmark Measurements for Organic Semiconductors. Org. Electron. 2014, 15, 1263-1272.

(39) Albrecht, S.; Tumbleston, J. R.; Janietz, S.; Dumsch, I.; Allard, S.; Scherf, U.; Ade, H.; Neher, D. Quantifying Charge Extraction in Organic Solar Cells: The Case of Fluorinated Pcpdtbt. J. Phys. Chem. Lett. 2014, 5, 1131-1138.

(40) Lenes, M.; Shelton, S. W.; Sieval, A. B.; Kronholm, D. F.; Hummelen, J. C.; Blom, P. W. M. Electron Trapping in Higher Adduct Fullerene-Based Solar Cells. Adv. Funct. Mater. 2009, 19, 3002-3007.

(41) Gann, E.; Young, A. T.; Collins, B. A.; Yan, H.; Nasiatka, J.; Padmore, H. A.; Ade, H.; Hexemer, A.; Wang, C. Soft X-Ray
Scattering Facility at the Advanced Light Source with Real-Time Data Processing and Analysis. Rev. Sci. Instrum. 2012, 83, 045110.

(42) Collins, B. A.; Li, Z.; Tumbleston, J. R.; Gann, E.; McNeill, C. $\mathrm{R}$; Ade, H. Absolute Measurement of Domain Composition and Nanoscale Size Distribution Explains Performance in Ptb7:Pc71bm Solar Cells. Adv. Energy Mater. 2013, 3, 65-74.

(43) Mukherjee, S.; Jiao, X.; Ade, H. Charge Creation and Recombination in Multi-Length Scale Polymer:Fullerene Bhj Solar Cell Morphologies. Adv. Energy Mater. 2016, 6, 1600699.

(44) Min, J.; Jiao, X.; Ata, I.; Osvet, A.; Ameri, T.; Bäuerle, P.; Ade, H.; Brabec, C. J. Time-Dependent Morphology Evolution of SolutionProcessed Small Molecule Solar Cells During Solvent Vapor Annealing. Adv. Energy Mater. 2016, 6, 1502579.

(45) Ye, L.; Jiao, X.; Zhang, S.; Yao, H.; Qin, Y.; Ade, H.; Hou, J. Control of Mesoscale Morphology and Photovoltaic Performance in Diketopyrrolopyrrole-Based Small Band Gap Terpolymers. Adv. Energy Mater. 2017, 7, 1601138.

(46) Kilcoyne, A. L. D.; Tyliszczak, T.; Steele, W. F.; Fakra, S.; Hitchcock, P.; Franck, K.; Anderson, E.; Harteneck, B.; Rightor, E. G.; Mitchell, G. E.; et al. Interferometer-Controlled Scanning Transmission X-Ray Microscopes at the Advanced Light Source. J. Synchrotron Radiat. 2003, 10, 125-136.

(47) Steyrleuthner, R.; Schubert, M.; Howard, I.; Klaumunzer, B.; Schilling, K.; Chen, Z. H.; Saalfrank, P.; Laquai, F.; Facchetti, A.; Neher, D. Aggregation in a High-Mobility N-Type Low-Bandgap Copolymer with Implications on Semicrystalline Morphology. J. Am. Chem. Soc. 2012, 134, 18303-18317.

(48) Mukherjee, S.; Proctor, C. M.; Tumbleston, J. R.; Bazan, G. C.; Nguyen, T.-Q.; Ade, H. Importance of Domain Purity and Molecular Packing in Efficient Solution-Processed Small-Molecule Solar Cells. Adv. Mater. 2015, 27, 1105-1111.

(49) Mukherjee, S.; Proctor, C. M.; Bazan, G. C.; Nguyen, T.-Q.; Ade, H. Significance of Average Domain Purity and Mixed Domains on the Photovoltaic Performance of High-Efficiency SolutionProcessed Small-Molecule Bhj Solar Cells. Adv. Energy Mater. 2015, 5,1500877 .

(50) Stolterfoht, M.; Armin, A.; Shoaee, S.; Kassal, I.; Burn, P.; Meredith, P. Slower Carriers Limit Charge Generation in Organic Semiconductor Light-Harvesting Systems. Nat. Commun. 2016, 7, 11944-11944.

(51) Jakowetz, A. C.; Böhm, M. L.; Zhang, J.; Sadhanala, A.; Huettner, S.; Bakulin, A. A.; Rao, A.; Friend, R. H. What Controls the Rate of Ultrafast Charge Transfer and Charge Separation Efficiency in Organic Photovoltaic Blends. J. Am. Chem. Soc. 2016, 138, 1167211679.

(52) Albrecht, S.; Schindler, W.; Kurpiers, J.; Kniepert, J.; Blakesley, J. C.; Dumsch, I.; Allard, S.; Fostiropoulos, K.; Scherf, U.; Neher, D. On the Field Dependence of Free Charge Carrier Generation and Recombination in Blends of Pcpdtbt/Pc70bm: Influence of Solvent Additives. J. Phys. Chem. Lett. 2012, 3, 640-645. 